\title{
Looking for the first time into the heart of the blazar TXS 2013+370
}

\author{
Thalia Traianou ${ }^{a}{ }^{*}$ Thomas P. Krichbaum $^{a}$, Bia Boccardi ${ }^{a}$, Emmanouil Angelakis ${ }^{a}$, \\ Roberto Angioni $^{a}$, Uwe Bach ${ }^{a}$, J. Anton Zensus ${ }^{a}$, Stefan Larsson ${ }^{b}$, Sebastian \\ KiehImann ${ }^{c}$, Mark Gurwell ${ }^{d}$, \\ ${ }^{a}$ : Max Planck Institute for Radio Astronomy, Auf dem Hügel 69, Bonn, Germany \\ ${ }^{b}:$ Department of Astronomy and Department of Physics, Stockholm University, AlbaNova, \\ SE-10691 Stockholm, Sweden and The Oskar Klein Centre for Cosmoparticle Physics, \\ Stockholm, Sweden \\ ${ }^{c}$ : Owens Valley Radio Observatory, California Institute of Technology, Pasadena, CA 91125, \\ USA \\ ${ }^{d}$ : Harvard-Smithsonian Center for Astrophysics, 60 Garden St, Cambridge, MA 02138, USA \\ E-mail: etraianou@mpifr.de
}

The compact radio source TXS $2013+370$ is a $\gamma$-ray blazar which is located at a redshift of $\mathrm{z}=$ 0.859 at a galactic latitude $b=1.2^{\circ}$. We observed the source with Very Long Baseline Interferometry (VLBI) at 15, 43 and $86 \mathrm{GHz}$ and studied the morphology and the kinematic properties of the jet. The VLBI data were then combined with flux density variability measurements at 15 and $235 \mathrm{GHz}$ and with the available $\gamma$-ray light curve in the period 2008-2017. A cross-correlation analysis was performed to investigate the existence of a correlation between the variability observed in the different bands. The preliminary results of our study showed that the most prominent flares and maxima stem from the central VLBI region and most likely are associated with the nuclear region, namely the core, indicating that the activity is caused by the passage of traveling shocks through the core region. In the course of our analysis, we present for the first time a $86 \mathrm{GHz}$ Global Millimeter VLBI Array (GMVA) image of the innermost jet region.

7th Fermi Symposium 2017

15-20 October 2017

Garmisch-Partenkirchen, Germany

${ }^{*}$ Member of the International Max Planck Research School for Astronomy and Astrophysics at the Universities of Bonn and Cologne. 


\section{Introduction}

Blazars are among the most powerful and variable known astrophysical objects. Their extreme properties are due to the existence of a relativistic jet of plasma ejected from the nuclear region and pointing towards the Earth at a very small viewing angle. This geometry give us the unique opportunity to investigate the structure and physical processes that take place in the innermost region of the jet. The $\gamma$-ray emitting blazar TXS $2013+370$ is such a compact object. It is located at a redshift of $z=0.859$ [ए]] and even though it is detected in $\gamma$-rays [ [ 4 ], it is not yet well studied due to its location close to the galactic plane $\left(b=1.2^{\circ}\right)$ [四]. By performing Very Long Baseline Interferometry (VLBI) observations at high frequencies, we can describe the morphology of the source, the kinematics on sub-parsec scales and probe how a change of the latter can contribute to the high-energy emission. The combination of VLBI kinematics data and flux density variability measurements in the radio/mm $/ \gamma$-rays often reveals correlations between them. The existence of such correlations can help us locate the $\gamma$-ray emission region and determine its distance from the central engine. For all calculations, we adopted the following cosmological parameters:

$\Omega_{M}=0.27, \Omega_{\Lambda}=0.23, H_{o}=71 \mathrm{~km} \mathrm{~s}^{-1} \mathrm{Mpc}^{-1}, \mathrm{D}_{L}=17.9$ Gly [Q] .

\section{Data Sample}

To trace the inner jet properties of the source, we used the inner jet VLBI observations at $43 \mathrm{GHz}$ and $86 \mathrm{GHz}$ with the Global Millimetre VLBI Array (GMVA) along with VLBI data from the MOJAVE program at $15 \mathrm{GHz}$ [Q]. We combined these with $\gamma$-ray data from Fermi Large Area Telescope (Fermi-LAT) ${ }^{1}$ and light curves at $15 \mathrm{GHz}$ from the Owens Valley Radio Observatory

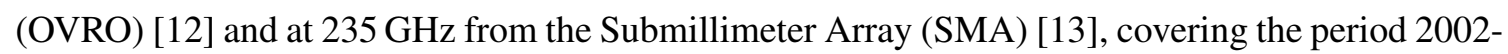
2017.

\section{Imaging and Kinematics Analysis}

We performed the imaging and model fitting of the VLBI data with the DIFMAP package using the CLEAN and MODELFIT algorithms respectively. The component cross-identification over 15 epochs at $15 \mathrm{GHz}$ and 5 epochs at 43 and $86 \mathrm{GHz}$ showed that the jet's brightness distribution can be expressed by 4 (at $15 \mathrm{GHz}$ ) and 3 (at $43,86 \mathrm{GHz}$ ) two-dimensional Gaussian components, which minimize the $\chi^{2}$ fitting value, as described in [6]. Errors in each parameter of the Gaussian components are calculated based on the approach of [[]]. A new component $(\mathrm{N})$ was ejected between October 2008 and March 2009, after found the core brighten by $80 \%$. The motion of the VLBI components is expressed by fitting polynomials to the radial separation from the core of each component (at each frequency) versus time. We find that a linear function is sufficient for fitting the trajectories of A1, A2 and C3. However, a second-order polynomial was needed to fit the trajectory of component C2 (Figure 1).

The linear and quadratic fit provide us an average proper motion, a mean speed and acceleration for each component. Using the estimated angular rate, $\mu$, we derived the apparent speed of each component to be: $\beta_{\text {appA1 }}=3.82 \pm 0.01, \beta_{a p p A 2}=6.76 \pm 0.37, \beta_{a p p C 2}=15.7 \pm 1.7$ and

\footnotetext{
${ }^{1}$ https://fermi.gsfc.nasa.gov/cgi-bin/ssc/LAT/LATDataQuery.cgi
} 
$\beta_{a p p C 3}=5.21 \pm 0.61$. Based on the apparent speed of the fastest component $\mathrm{C} 2$ and following the method of []] we determined the critical viewing angle of the jet to be $\vartheta_{c r i t}=3.65^{\circ}$.
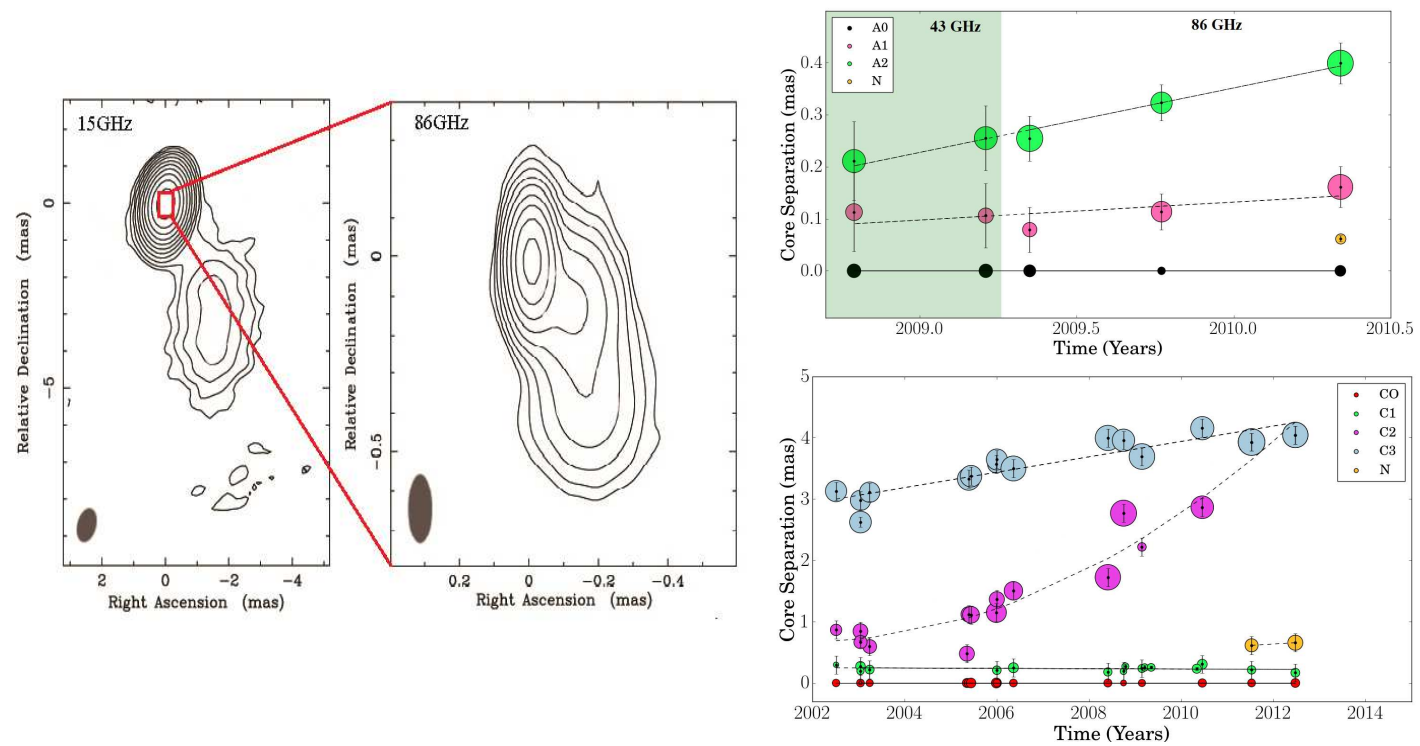

Figure 1: Left panel: A synthesis of a $15 \mathrm{GHz}$ image of TXS $2013+370$ (beam size $0.9 \times 0.6$ mas at $-17.8^{\circ}$ ) and at the $86 \mathrm{GHz}$ (beam size $0.18 \times 0.07$ mas at $0^{\circ}$ ). Top right: The core separation of the fitted jet components versus time at $43 \mathrm{GHz}$ and $86 \mathrm{GHz}$. A new component, labeled N, is visible in May 2010. Bottom right: Components radial separation from the core versus time at $15 \mathrm{GHz}$ during period 2002-2012.

Particularly interesting is the case of knot $\mathrm{C} 1$ which appears quasi-stationary, but there are some indications that it oscillates around a mean position. Bending jets often present quasistationary shocks [[]]. Numerous studies have shown that when a moving shock passes through a stationary one, the latter could be displaced in position for a short time and then return to its initial position [ [8]. In our case, components $\mathrm{C} 1$ and $\mathrm{A} 2$ may exhibit such behaviour. Another possible explanation is that we observe some motion of the $\tau=1$ opacity surface that we use as a reference point, after the ejection of a new component. In this scenario, when the core moves upstream (towards the supermassive black hole) the distance of all secondary components slightly increases, until the new component is well separated and the core moves back to its initial position. More observations are needed in order to explain the path of the $\mathrm{C} 1$ component.

\section{Light Curve Analysis}

We searched for possible correlations between the radio and $\gamma$-ray variability. Using the Discrete Cross-Correlation Function (DCCF) we found evidence for a positive correlation between the $\gamma$-rays and $235 \mathrm{GHz}$ lightcurve. The DCCF analysis [B] revealed that the $235 \mathrm{GHz}$ radio activity lags behind the $\gamma$-rays by $91 \pm 24$ days and between $\gamma$-rays and $15 \mathrm{GHz}$, also radio reach our antennas $103 \pm 30$ days after the high energy activity. This indicates that the gamma-ray emission originates in the region upstream of the compact VLBI core. The time delays can be converted to linear distances between the regions of the radio synchrotron opacity barrier and the $\gamma$-ray emission region $[\square]$. Converting the aforementioned time lags into linear distances, we determine the 
distance between the $\gamma$-ray production region and the VLBI core to be $(10.1 \pm 2.7) \mathrm{pc}$ at $86 \mathrm{GHz}$ and $(11.6 \pm 3.3) \mathrm{pc}$ at $15 \mathrm{GHz}$.

\section{Conclusions}

In this work, we present for the first time $86 \mathrm{GHz}$ VLBI images of the inner structure of the blazar TXS $2013+370$. Based on multi-band variability light curves our preliminary results revealed a correlation between the gamma-ray and radio variability at $86 \mathrm{GHz}$ and $15 \mathrm{GHz}$. We have cross-identified superluminally moving knots on subparsec scales and we report the ejection of a new component, which is associated with prominent flare events in the millimeter and high energy bands. Tracing the peculiar motion of the jet component $\mathrm{C} 1$ and overlaying it with $\mathrm{A} 2$, we conclude that it is a shock, which first moves downstream and then returns back to its original position. We created for the first time high resolution images of the source at $86 \mathrm{GHz}$ and $43 \mathrm{GHz}$. Our variability analysis showed that the high energy activity is strongly correlated with the $15 \mathrm{GHz}$ and $235 \mathrm{GHz}$ variability, with radio lagging behind $\gamma$-rays. This behaviour is consistent with synchrotron selfabsorption in the VLBI jet and suggests that the $\gamma$-rays stem from a region which is located about $10 \mathrm{pc}$ upstream to the VLBI core.

\section{References}

[1] A. A. Abdo, et al., Fermi Large Area Telescope First Source Catalog, 2010, ApJ, 188, 405, [arXiv:1002.2280]

[2] A. Alberdi, et al.,The Parsec-Scale Radio Jet of 4C 39.25, 1993, ApJ, 402, 160

[3] L. Fuhrmann, et al., Detection of significant $\mathrm{cm}$ to sub-mm band radio and $\gamma$-ray correlated variability in Fermi bright blazars, 2014, MNRAS, 441, 1899, [arXiv:1403.4170]

[4] E. Kara, et al., Gamma-Ray Emission from Two Blazars Behind the Galactic Plane: B2013+370 and B2023+336, 2012, ApJ, 746, 2, [arXiv:1112.3312]

[5] V. Karamanavis, et al.,PKS 1502+106: A high-redshift Fermi blazar at extreme angular resolution. Structural dynamics with VLBI imaging up to $86 \mathrm{GHz}, 2016$, A\&A 586, A60, [arXiv:1511.01085]

[6] T. P. Krichbaum, et al, VLBI observations of Cygnus A with sub-milliarcsecond resolution, 1998, A\&A, 329,873

[7] J. Leon-Tavares, et al., The connection between the gamma-ray emission and millimeter flares in Fermi/LAT blazars, 2011, A\&A 532, A146, [arXiv:1110.5267]

[8] B. Rani, et al.,Connection between inner jet kinematics and broadband flux variability in the $B L$ Lacertae object S5 0716+714, 2015, A\&A 578, A123, [arXiv:1503.04218]

[9] M. L. Lister, et al.,MOJAVE: Monitoring of Jets in Active Galactic Nuclei with VLBA Experiments. V. Multi-Epoch VLBA Images, 2009, AJ, 137, 3718, [arXiv:0812.3947]

[10] M. S. Shaw, et al., Spectroscopy of the Largest Ever Gamma-Ray-selected BL Lac Sample, 2013, ApJ, 764, 13, [arXiv:1301.0323]

[11] R. C. Vermeulen \& M. H. Cohen, Superluminal motion statistics and cosmology, 2014, ApJ, 430, 467

[12] J. L. Richards, et al., Blazars in the Fermi Era: The OVRO $40 \mathrm{~m}$ Telescope Monitoring Program, 2011, ApJS, 194, 29, [arXiv:1011.3111] 
[13] M. A. Gurwell, et al., Monitoring Phase Calibrators at Submillimeter Wavelengths, 2007, From Z-Machines to ALMA: (Sub)Millimeter Spectroscopy of Galaxies, eds. A. J. Baker, J. Glenn, A. I. Harris, J. G. Mangum, \& M. S. Yun, ASP Conf. Ser., 375, 234,

(http://aspbooks.org/custom/publications/paper/375-0234.html) 